\title{
Preparation Process and Quality Inspection of Coriolan Oral Liquid
}

\author{
Xiuying Wang ${ }^{1, a}$, Wei Zhang ${ }^{2, b}$, Chunlin Zhao ${ }^{1, c}$, Xue Xiao ${ }^{1, d}$ \\ ${ }^{1}$ College of Traditional Chinese Medicine, Jilin Agriculture Science and Technology College, Jilin, \\ 132101, China \\ ${ }^{2}$ Changchun Beihua Pharmaceutical Co., Ltd., Changchun, 130021, China \\ aemail, ${ }^{b}$ email, ${ }^{\mathrm{c}}$ email, ${ }^{\mathrm{d}}$ email
}

Keywords: Coriolan oral liquid, Preparation process, Quality inspection, Quality standards

\begin{abstract}
The best process was determined through determination in different temperature of polysaccharide completely dissolved time, the precipitation coefficient after standing some of the different time and the filtering effect of different filter medium. The Coriolan oral liquid was trial produce by the best technology and its quality was tested. The result was showed that the best dissolved time of polysaccharide was $80{ }^{\circ} \mathrm{C}$, and the best standing time was $24 \mathrm{~h}$ of polysaccharide solution, and the best filter medium was a layer of Cotton wool. The result of quality test was in line with national standards about density, $\mathrm{pH}$ value, color reaction, polysaccharide content and microorganism content. The final quality level of Coriolan oral liquid was that properties was brown liquid and the tast was sweet, while relative density was 1.048-1.058, but pH value was 6.7-6.9 and the content of polysaccharide of Coriolan was 32.86-33.21.
\end{abstract}

Coriolus is extracted from Polystictus versi-color (L.) protein polysaccharide extracted by Fr., mainly composed of dextran, and contains 5 kinds of other types of sugar (xylose, galactose, mannose, in Li Tang, Arabia, sugar) from mycelia cultured in the body can also be isolated from Coriolus versicolor polysaccharide. Since 1965, Japanese scientists began to introduce polysaccharide anti-tumor research field has been a full 40 years of history, modern pharmacological research shows that polysaccharide has significant anti-tumor, anti-ulcer, liver protection, antiviral, hypolipidemic and immune regulation for. PSK products currently on the market mainly for granules. In 1980s, it is widely in the country when the outbreak of hepatitis A. Since the absorption of the tablets is slower after the oral administration, the granules are affected by certain environmental factors. Therefore, the oral liquid is changed into oral liquid so as to promote absorption, convenient drinking and enhancing curative effect.

\section{Materials, Reagent and Instrument}

Experimental Materials. Wild Coriolus versicolor (purchased in Jilin City, identified as Coriolus versicolor by the resource identification laboratory of Agriculture Science and Technology College), dried and crushed, screened with 60 meshes.

Reagent and Instrument. JYT-2 drug balance (Shanghai medical laser instrument factory); AL-204 electronic balance (Mettler Toledo (Shanghai) Co., Ltd.); WMZK-72 type pointer type electric thermostatic water bath (Shanghai Yuejin Medical Instrument Factory); DHG-9035A type electro thermal blowing dry box (Shanghai source long experimental equipment factory); type SY-360 (ultrasonic extractor Shanghai Ning bounce Instrument Co. Ltd.); taking UV-1700PharmaSpec type ultraviolet visible spectrophotometer (Beijing Puxi pass Instrument Co. Ltd.); YXQ-LS-50S type steam sterilizer (Shanghai Moldova billion); XCQ-V type ultrasonic wave bottle washing machine (Jining Hengda ultrasonic equipment Co. Ltd.); GMSU-054W type tunnel dryer (Shanghai xufa Pharmaceutical Machinery Co., Ltd.); sealing machine (YGE); bottle dryer (LGQX- III); light inspection machine (DGT). Shanghai Dongfeng cellulase Biochemical 
Technology Co., 98\% sulfuric acid, 5\% phenol, anhydrous ethanol, purified water, aspartame, edible essence, alkaline copper tartrate solution, anhydrous glucose control.

\section{Experimental Method}

Extraction of Coriolus. Coriolus versicolor (60 mesh), crushing 95\% ethanol degreasing (refluxing 3 times), 20 min $55 \mathrm{C}$ enzyme (cellulase concentration 1.5\%, pH6.0), the ratio of material to liquid 1:25, 20 min $45 \mathrm{C}$ ultrasonic extraction, the extraction rate of polysaccharide was $4.34 \%$, drying for standby.

Optimization of Dissolution, Standing and Filtering. Weigh the polysaccharide $72 \mathrm{~g}$ added $2000 \mathrm{ml}$ versicolor in purified water (12) 20, 40 constant temperature water bath at a temperature of 80 DEG C, 60, dissolved, to determine the optimum temperature when the sugar dissolved. Many sugar dissolved packaging 500mL conical flask static observation, precipitation, drying and weighing, determine the best holding time. After 24 hours, the polysaccharide solution is filtered. The filter media are: medium speed filter paper, fast filter paper, two layers of medical gauze, four layers of medical gauze, one layer of absorbent cotton and two layer of absorbent cotton. Take 2000mL polysaccharide solution, use different filter medium to filter.

Preparation and Quality Test of Coriolus Oral Liquid. The content in the oral liquid is determined according to the content of yunzhigantai granules of polysaccharide krestein. Containing polysaccharide $0.33 \mathrm{~g} /$, each $10 \mathrm{ml}$, aspartame 3 per thousand, 1 per thousand flavor. Vertical steam sterilizer (YXQ-LS-50S) set at a temperature of 121, sterilization time is 21min, the gland after polysaccharide oral liquid were sterilized 2 times. Observation of polysaccharide oral liquid product color, taste the taste of Coriolus versicolor polysaccharide. Take clean, dry and precise weighing bottle, filled with the sample (lower than $20 \mathrm{C}$ ), with the center of the pores of the bottle, the bottle stopper overflow liquid with filter paper dried, placed 20min in 20 DEG C, with constant temperature water bath, the test solution temperature rise, liquid will continue from the mouth overflow, using filter paper wipe the liquid to liquid overflow, overflow will no longer remove the bottle. Dry the liquid outside the weighing bottle and weigh it accurately. Calculate the weight of the sample. Pour the sample out, wash it, fill the newly boiled cold water, and then measure the weight of the water (20 centigrade). Determination of polysaccharide content in samples from each batch products: $1 \mathrm{ml}, 5 \mathrm{~mL}$ volumetric flask, water to volume, and then take the bottle from the quantitative $2 \mathrm{ml}$ in a test tube, and then the same method and standard curve of the absorbance was measured, the calculation of polysaccharide concentration according to the linear regression equation.

\section{Results and Analysis}

Dissolution Temperature Screening of Coriolus Powder. The data of Figure 1 show that the constant temperature of 80 DEG $\mathrm{C}$ water bath stirring with a glass rod, the dissolution rate of polysaccharide is the fastest, but considering the energy consumption, choose $60 \mathrm{C}$ dissolution time was $12 \mathrm{~min}$, the best solution temperature is more appropriate.

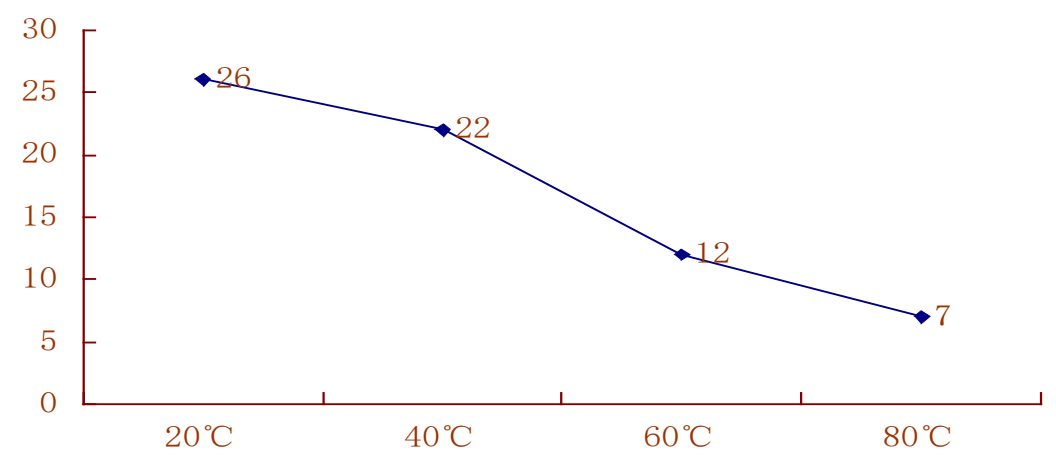

Figure 1. Dissolution time of Coriolus Powder of different temperatures 
Standing Time Optimization. The data of Table1 shows that the static $24 \mathrm{~h}$ and $32 \mathrm{H}$ precipitation of $1.213 \mathrm{~g}$ and $1.226 \mathrm{~g}$, for oral liquid by adding polysaccharide solid substances in $6.74 \%$ and $6.81 \%$, both in solution and $32 \mathrm{H}$ thin layer of uniform sediment, static solution precipitation had no obvious increase, considering the actual production efficiency.

Table 1. Precipitation weight of different standing time

\begin{tabular}{|c|c|c|c|c|}
\hline \multirow{2}{*}{} & \multicolumn{4}{|c|}{ Standing time (h) } \\
\cline { 2 - 5 } & $8 \mathrm{~h}$ & $16 \mathrm{~h}$ & $24 \mathrm{~h}$ & $32 \mathrm{~h}$ \\
\hline Precipitation (g) & 0.512 & 0.832 & 1.213 & 1.226 \\
\hline $\begin{array}{c}\text { Ratio of ganoderma } \\
\text { polysaccharides (\%) }\end{array}$ & 2.84 & 4.46 & 6.74 & 6.81 \\
\hline Precipitation state & Fine precipitation & $\begin{array}{c}\text { Homogeneous } \\
\text { particle } \\
\text { precipitation }\end{array}$ & $\begin{array}{c}\text { Homogeneous } \\
\text { thin-layer } \\
\text { precipitation }\end{array}$ & $\begin{array}{c}\text { Homogeneous } \\
\text { thin-layer } \\
\text { precipitation }\end{array}$ \\
\hline
\end{tabular}

Filtration Medium Optimization. The data of Table 2 shows that better filter effect, but a long time, and the use of a cotton filtering speed is relatively fast, the filtrate clarity is good, from a small amount of precipitation that rocked the scattered, meet the quality standard of oral liquid, comprehensive evaluation, selection of a layer of cotton as a filter medium.

Table 2. Filtration results of different filtration mediums

\begin{tabular}{|c|c|c|}
\hline Filtration Medium & Filtration time (min) & Filtrate clarification \\
\hline Medium Filter paper & 240 & Clarification \\
\hline Fast Filter paper & 190 & Clarification \\
\hline Two layers of medical gauze & 35 & Turbidity \\
\hline Four layers of medical gauze & 65 & Turbidity \\
\hline One layer of absorbent cotton & 95 & A little precipitation \\
\hline Two layers of absorbent cotton & 140 & Micro precipitation \\
\hline
\end{tabular}

Characteristic Identification. A total of 6 batches of products tested (Table 3), including 02, 04, 05, 06, four batches of brown color, 01 color light, 03 color deep, comprehensive comparison to determine the tan, sweet for quality evaluation of polysaccharide oral liquid appearance.

Table 3. Characteristic identification of oral liquid of different product batch numbers

\begin{tabular}{|c|c|c|}
\hline Product batch number & Color & Texture \\
\hline 1 & Light brown & Sweet \\
\hline 2 & Chocolate-brown & Sweet \\
\hline 3 & Dark brown & Sweet \\
\hline 4 & Chocolate-brown & Sweet \\
\hline 5 & Chocolate-brown & Sweet \\
\hline 6 & Chocolate-brown & Sweet \\
\hline
\end{tabular}

Identifications of Relative Density, PH Value and Color. According to the national pharmacopoeia, the density should be 1.03 - 1.06; the $\mathrm{pH}$ value should be 6-7; precipitation of red cuprous oxide should be precipitated in the solution. Table 3-4 data showed that 6 batches of oral liquid color identification examination and $\mathrm{pH}$ value test results were qualified, of which 02 batches of relative density is slightly lower than the standard, 03 batches of relative density higher than the standard provisions, the rest are in the acceptable range. 
Table 4. Testing results of physical characteristics of oral liquid of different product batch numbers

\begin{tabular}{|c|c|c|c|}
\hline Product batch number & Relative density & $\mathrm{pH}$ Value & Color identification \\
\hline 1 & 1.035 & 6.8 & Red precipitation \\
\hline 2 & 1.019 & 6.7 & Red precipitation \\
\hline 3 & 1.079 & 6.7 & Red precipitation \\
\hline 4 & 1.058 & 6.9 & Red precipitation \\
\hline 5 & 1.056 & 6.7 & Red precipitation \\
\hline 6 & 1.048 & 6.8 & Red precipitation \\
\hline
\end{tabular}

Microbial Limit. In the 2010 edition of the Pharmacopoeia of China, the number of bacteria in each $1 \mathrm{~mL}$ sample shall not exceed 100, the number of moulds and yeasts should not exceed 10, and Escherichia coli should not be detected. Table 3-5 data showed that sterilization was carried out at a temperature of 121 DEG C and sterilization time of $21 \mathrm{~min}$, and the results of sterilization were satisfactory. The microbiological examination of 6 batches of products was in line with the regulations.

Table 5. Microbial limit test

\begin{tabular}{|c|c|c|c|}
\hline \multirow{2}{*}{$\begin{array}{c}\text { Product batch } \\
\text { number }\end{array}$} & $\begin{array}{c}\text { Number of mould and } \\
\text { yeast }\end{array}$ & Number of bacteria & Escherichia coli \\
\cline { 2 - 4 } & cells/ml & cells/ml & cells/ml \\
\hline 1 & 3 & 30 & 0 \\
\hline 2 & 4 & 34 & 0 \\
\hline 3 & 7 & 51 & 0 \\
\hline 4 & 8 & 49 & 0 \\
\hline 5 & 6 & 40 & 0 \\
\hline 6 & 5 & 39 & 0 \\
\hline
\end{tabular}

\section{Conclusion}

This study mainly yunzhigantai granules as the prototype for the improvement of dosage form preparation, according to oral liquid, screening and identification of the main process of polysaccharide oral liquid, polysaccharide oral liquid on the preparation of the quality testing, set up the quality standard of Coriolus Versicolor Polysaccharide oral liquid.

The filtering effect under different temperature conditions of polysaccharide solution, dissolving time of static time sedimentation rate, different filter media to determine the best process. The optimum polysaccharide dissolution temperature was 60 DEG C, the optimum retention time was 24h, and the optimum filter medium was single layer absorbent cotton.

Comparison of the quality test results, finally establish the quality standard of Coriolus Versicolor Polysaccharide oral liquid. Product character identification required color is brown and sweet; measured the relative density of 1.048-1.058, close to 1.058 of the national standard limit, easy to appear in the process of mass production of substandard products, so as to require less than 1.058; the $\mathrm{pH}$ value of 6.7-6.9; color discrimination reaction solution in red cuprous oxide precipitation; polysaccharide content determination 32.86-33.21mg.mL-1, in mass production can be reduced by 20\%; microbial limit requirements in line with national standards.

In summary, a successful trial of the oral liquid, to find out the process route for mass production, but also to complete the quality standard of Coriolus versicolor oral liquid formulation, provide data basis for enterprise production. 


\section{References}

[1 Li Caidong, Wu Bin, Shi Yanping, Study on the Quality Standard of Gantai Tablets [J]. Lishizhen Medicine and Materia Medica Research, ,2008,19(11): 2736-2737.

[2] Dong Xin, Song Feng, Yu Xiurong, Qualification Monitoring Techniques and Methods of Steam Sterilizer [J]. Chinese Journal of Nosocomiology, 2009,19(15): 1988-1989.

[3] Dong Wenhui, Xiao Chune, Sha Yingwei, Liang Chunhua, Spectrophotometric Determination of the Polysaccharides from Polystictus Versicolor (L) FR. In Yunzhi Gantai Granules [J]. Journal of China Pharmaceutical University, ,1989,20(3): 175-178.

[4] Wang Feifei, Hao Limin, Jia Shiru, Chen Qiang, Niu Shuang, Review on Poly Saccharide from Coriolus Versiclor [J]. Food and Fermentation Industries, 2012,38(6): 148-152. 